\title{
The Principle and Application of the Multi-Axis Linkage Machining Center
}

\author{
Qinghui Dai ${ }^{1, a}$, Sisi Ren ${ }^{1, b}$ \\ ${ }^{1}$ Department of Mechanical Engineering, North China Electric Power University, Baoding, Hebei \\ 071003, China \\ adcba6789@126.com, bbitter_lemon66@163.com
}

Keywords: Multi-axis linkage, Principle, Blade machining.

\begin{abstract}
With the progress of science and technology, product parts machining processes are more and more difficult, and the machining accuracy is becoming higher and higher. The application of multi-axis linkage machining center is the main way to solve these problems. As a major means to solve special-shaped complex, which contains complex curved surface and crank shaft and so on, five-axis linkage machining center is very representative in multi-axis linkage machining center. Through the application of using five-axis linkage machining center to process the blade, the conclusions are that the quality and efficiency of five-axis linkage machining center have been improved compared with the three-axis linkage and four-axis linkage machining center, and the necessity and feasibility of the development of multi-axis linkage machining center are drew.
\end{abstract}

\section{Introduction}

Machine tool is the symbol of a country's manufacturing level. Modern NC machining is developing towards higher speed, precision, intelligence, flexibility, automation and reliability. Multi-axis NC machine tools reflect the above points. Multi-axis linkage machining center is that numerical control system controls multiple axes to move coordinately and synchronously according to the requirements of functional relation. According to the number of axes, numerical control machines are divided into two-axis linkage, two and a half axes linkage, triaxial linkage, four-axis linkage, five- axis linkage, etc. As the design complexity of workpieces is higher and higher, the use of triaxial linkage and four-axis linkage can't meet the demand of many complex special-shaped products.

Wei Fang et al. had been stimulated four-axis machining processing and then compared with five-axis machining center in cutting speed and cutting state. They found that the efficiency of five-axis linkage machining center was increased greatly [1]. Hao Chen et al. thought six axes linkage machining improved the processing flexibility and freedom of motion greatly, this system can adapt to machining more types of workpieces and more complex curved surfaces [2]. In October 2014, China Transmission Heavy Duty Machine tool co., LTD developed a machine tool independently, which is for processing the propellers, with seven axes of six linkages to response to the customer's requirements, and the machine worked very well. It had the largest processing diameter and was the most complex machine tool around the world [3]. Compared with a five-axis linkage machining center, its machining precision and machining efficiency were improved.

Machining efficiency and machining precision are improved through multi axes that more than five linkage machining center, but they are more strict with professionals and technology, and also increase the processing cost, narrow the adaptations; Triaxial linkage and four-axis linkage machining center cost lower, however, they can't reach the requirement of such precision and efficiency and can't achieve complex curved surface machining. As a result, the demand of five-axis linkage machining center have been promoted in China in resent years.

Though five-axis linkage machining center is not the most advanced multi-axis linkage machining center currently, it has a typical representative of the meaning, and has been widely applied at present. 
Five-axis linkage CNC technology is also a symbol of a country's industrialization level across the world.

\section{The Principle of Multi-Axis Linkage Machining Center}

Multi-axis NC machining researches mainly include three aspects: (1) physical process simulation of machining and product quality forecasting, (2) dynamic response of process system and stability analysis, (3) efficient NC machining process planning. Five-axis linkage machining center is typical at all researching aspects of multi-axis linkage machining center, because it represents the highest state of the machine tool manufacturing industry at present. Detailed introductions of five-axis linkage machining center's principles are as follows.

The Structure of the Machining Center. Most five-axis linkage machining centers are the structure of $3+2$. There are three straight axes and two of the three ratate as the rotation axes. According to the three straight axes and the combination of two of three rotation axes, five-axis linkage machining center has three types, they are X, Y, Z, A, B (Fig. 1); X, Y, Z, A, C; X, Y, Z, B, C. By the form of the combination of the two axes of rotation, there are three kinds of forms: double rotating table type, turntable oscillating type and double pendulum type [4]. Because of physical reasons, the size of the specifications of the machine tool and the machining range of objects are decided by the three kinds of structure forms.

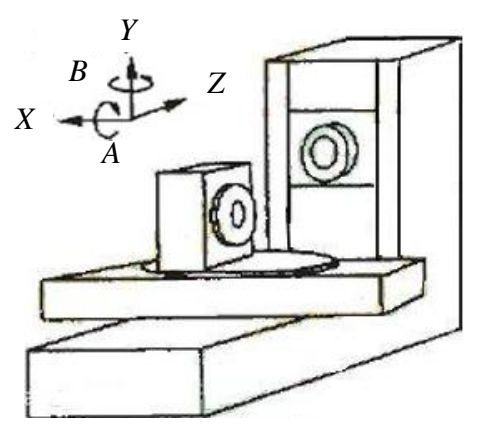

Fig. 1 The principle of horizontal five-axis linkage machining center

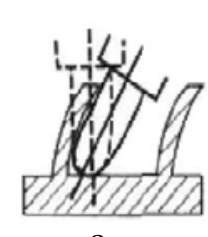

a

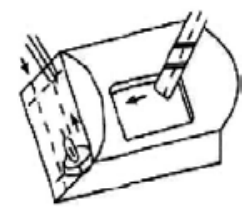

d
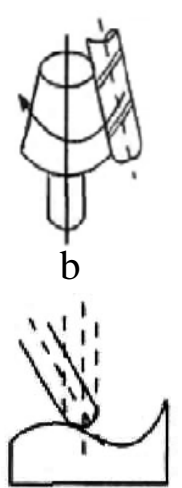

e
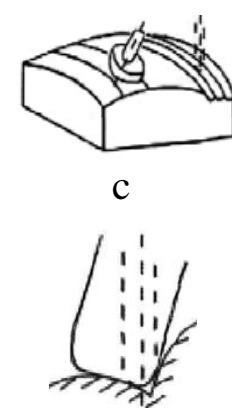

$\mathrm{f}$
Fig. 2 The characteristics of five-axis linkage machining

The Characteristics of the Machining Center. The characteristics of five-axis linkage machining center are shown in Fig. 2: (a) It can avoid cutter interference, and have wide adaptability and process complex parts which is difficult for triaxial linkage machining center. (b) For ruled surface parts, it can use flank milling to processing by a formation shape in good quality and high efficiency. (c) As for general multiple-layered surfaces, especially for relatively large flat surfaces, it is available to use large diameter milling cutter to approach the surface. In this way, the feeding of the cutter is fewer; the residual height of the workpiece is smaller. It improves the machining efficiency and surface quality greatly. (d) The benefit of five-axis machining center is having the ability to machine complex shapes in a single set-up. It is helpful to improve the positional precision of the surface and to gain high efficiency. (e) When five-axis machining center works, the cutter can be in the most efficient cutting state to the workpiece's surface relatively. (f) Under certain machining conditions, it's good to use large size of cutting tool to avoid the interference, and the cutters are in good condition of rigidity at the same time, this method improves the machining efficiency and precision.

The CNC System of Machining Center. It is the brain of multi-axis machining center, and also the main element to decide functionality and performance of the machining center. Numerical control system researches mainly include three aspects: (1) fine interpolation of multi-axis NC machining, (2) interaction of equipment and process, (3) closed loop control of NC machining process. 
Essentially speaking, based on the input of the numerical control procedures, five-axis linkage machining center CNC system interpolates the ideal trajectory, and then outputs the orders to the executive parts of the machining center to machining workpieces that needed. The basic function of the NC system includes 5 modules: the input, the trajectory interpolation, the servo control, the feedback control and the automatic tool change. It's an enclosed control system with position control as well. To ensure that the operating components are on schedule, the feedback control system tracks the performance of the implementation of the measurement in real time, and puts the feedback of the possibly error signals to the control system, then combines those possibly error signals with the next input signals as the output signals to executive parts.

The high-grade CNC system should meet the following basic elements [4]: (1) the multi-channel and multi-axis linkage; (2) the functions of space tool compensation and optimization; (3) full or double closed-loop control ability; (4) interpolation period $<0.5 \mathrm{~ms}$; (5) predictive control $>1000$ procedures section; (6) data operation and switching unit $<\mathrm{lnm}$.

\section{The Application of Multi-Axis Linkage Machining Center}

Multi-axis linkage machining center can solve spatial arbitrary curved surface along the surface normal, spatial twisted curved surface machining interference and turning parts processing. The application of multi-axis linkage machining center is very widely, covering ship manufacturing, aerospace and other industrial fields. In terms of turbine blade, because of the complex curved surface, certain bending and distortion degree, Machining blade is difficult but also important.

The five-axis linkage machining center can solve the problems of impact between the blade and the cutter [5], and ensure the process surface without overcut and dead angles when the machine processing the blade.

A five-axis linkage machining center of turbine blade SMC40160 is shown in Fig. 3 [6]. This five-axis linkage machining center is equipped with Openmind Company's professional programming software Hypermill. The machining center is composed of straight axes and rotating shafts. Straight axes are the $\mathrm{X}, \mathrm{Y}, \mathrm{Z}$. Rotating shafts are the blade rotating shaft $\mathrm{A}$ and the tool swinging shaft $\mathrm{B}$.
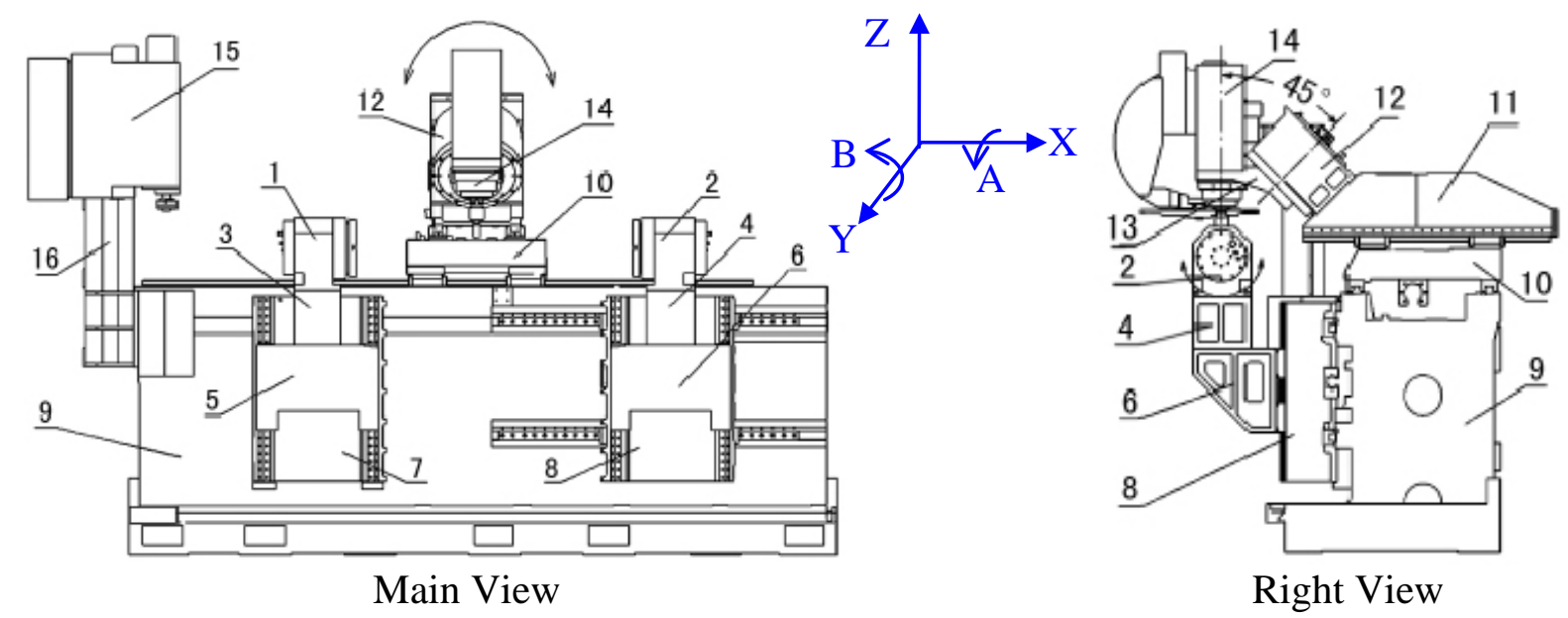

Fig. 3 The structure of five axis machining center

1-head 2-the tail 3-head frame base 4 -end bracket base 5 - $\mathrm{Z}_{1}$ axis drag plate

$6-\mathrm{Z}_{2}$ axis drag plate $7-\mathrm{Z}$ axis base $8-\mathrm{X}_{2}$ axis drag plate 9 -bed $10-\mathrm{X}_{1}$ shaft dragging plate

11-Y dragging plate $12-\mathrm{B}$ axis 13 -stent 14 -milling spindle 15 -knife library

16-knife library supporter

In this machining center, the routes of the $\mathrm{Y}$ axis and the $\mathrm{Z}$ axis are shorter than usual; the $\mathrm{X}$ axis is longer in order to adjust the length of the blade. The purpose of the $X_{2}$ drag plate is to adjust the distance between the $Z_{1}$ and $Z_{2}$ to adapt to the length of the blade, it's not moving at the time of 
processing; $\mathrm{Z}_{1}$ drag plate and $\mathrm{Z}_{2}$ drag plate clamp blade and move in a linear motion with the same direction and the same rate at the same time; A shaft and vice-A shaft, which combined as one as the rotating shaft, rotate at the same time the same direction and the same rate, as a result, the pulling force is produced, the machining precision is improved and the distortion is decreased; In order to maintain the standard of processing range and avoid the influence of torque on the rigidity of the $B$ axis, the angle between the $\mathrm{B}$ axis and the horizontal is $45^{\circ}$, the rotation range of the $\mathrm{B}$ axis is $\pm 50^{\circ}$.

Through the case analysis, the five-axis linkage machining center has complex structure and needs professional programming software badly. From the point of the result, the five-axis linkage machining center leads to better quality and efficiency than four-axis linkage and three-axis linkage machining center. From this point of view, the more the machine axes are, the more complicated the parts is, and the higher the machining precision is. Along with the development of technology, five-axis linkage machining center is no longer the most advanced multi-axis linkage machining center. More and more multi-axis linkage machining center need to be developed to adapt to wider range of requirements.

\section{Conclusions}

In order to design and develop the multi-axis machining center for complex parts, the principle of the typical five-axis machining center is set to be the foundation. The principle of five-axis linkage machining center was introduced in detail, and example is shown to deepen the explanation. In application example, the basic structure of the five-axis linkage machining center had no obvious changes, and its processing results showed that the machining quality and efficiency have improved distinctly, which means that five-axis linkage machining center has the strong flexibility and superiority in machining processing.

The principle of five-axis linkage machining center is typical to multi-axis linkage machining center and its adaptability is strong enough. However, with the complexity of the parts becoming higher and higher, five-axis linkage machining center can't meet all the requirements. Multi-axis linkage CNC machine tools are the concentrated expression of variety process of numerical control technology, and they should meet all kinds of complicated surface parts processing. To strengthen the power of manufacturing, it is necessary to develop more than five axes multi-axis linkage machining center.

With the number of axes for linkage increasing, however, the cost will be higher, the structure will be more complicated, and the technician requirements will be more stringent, the software programming will be more difficult. All of those are the key problems for application of multi-axis linkage machining center, and scientists and technicians are motivated by those problems.

\section{References}

[1] C. Weifang, C. Juite, Jiahong S: Key Engineering Materials Vol. 625 (2015), p. 241.

[2] Hao Chen, Mo Chen, Xuecheng Xi, Wansheng Zhao: Electromachining \& Mould No. 6 (2013), p. 70. In Chinese.

[3] Information on http://news.163.com/14/1028/13/A9L98DTH00014JB6.html

[4] Yuxian Du, Qijian Lu, Mingdeng Liu: Machinery Manufacturing \& Automation No. 3 (2008), p. 14. In Chinese.

[5] H.T. Young, L.C. Chuang, K. Gerschwiler, S. Kamps: International Journal of Advanced Manufacturing Technology Vol. 23(3-4) (2004), p. 233

[6] Xiaolei Li, Xiangzhi Kong, Kehui Yan, Zhixin Wang, Xu Ma: Mechanical Research \& Application No. 5 (2011), p. 113. In Chinese. 